\title{
Design and Development of Anti-Icing Aluminum Surface
}

\author{
Yongxin Wang ${ }^{1}$, Daniel Orol ${ }^{2}$, Jeffery Owens ${ }^{3}$, Katherine Simpson ${ }^{3}$, Hoon Joo Lee ${ }^{1}$ \\ ${ }^{1}$ Textile Engineering, Chemistry and Science, North Carolina State University, Raleigh, USA; ${ }^{2}$ Cary Academy, Cary, USA; ${ }^{3}$ Air \\ Force Research Laboratory, Tyndall Air Force Base, Tyndall, USA. \\ Email: hoonjoo_lee@ncsu.edu
}

Received April $9^{\text {th }}, 2013$; revised May $8^{\text {th }}, 2013$; accepted May $19^{\text {th }}, 2013$

Copyright (C) 2013 Yongxin Wang et al. This is an open access article distributed under the Creative Commons Attribution License, which permits unrestricted use, distribution, and reproduction in any medium, provided the original work is properly cited.

\begin{abstract}
An anti-icing surface has been designed and prepared with an aluminum panel by creating an artificial lotus leaf which is highly hydrophobic. The hydrophobicity of a solid surface can be generated by decreasing its surface tension and increasing the roughness of the surface. On a highly hydrophobic surface, water has a high contact angle and it can easily rolls off, carrying surface dirt and debris with it. Super-cooled water or freezing rain can also run off this highly hydrophobic surface instead of forming ice on the surface, due to the reduction of the liquid-solid adhesion. This property can also help a surface to get rid of the ice after the water becomes frozen. In this study, a Cassie-Baxter rough surface was modeled, and an aluminum panel was physically and chemically modified based on the modeled structure. Good agreement was found between predicted values and experimental results for the contact and roll-off angles of water. Most importantly, by creating this highly hydrophobic aluminum rough surface, the anti-icing and de-icing properties of the modified surface were drastically improved compared to the control aluminum surface, and the cost will be reduced.
\end{abstract}

Keywords: Anti-Icing; De-Icing; Wetting; Hydrophobic; Aluminum

\section{Introduction}

The phenomenon that ice builds up on the surfaces of a plane could cause significant problems to the aircrafts, such as lift and drag; especially, accumulations of ice could result in severe transportation problem due to the imbalances of the aircrafts under a high flying speed [1]. Lynch and Khodadoust found that various types of ice accretions on surfaces of fixed wing aircraft can affect the aerodynamic performance, maneuverability and control degradations of an aircraft, and even a small amount of ice accretions at critical locations could cause substantial problems, such as reduction of engine performance and its stability [2]. For a flying aircraft, freezing rain and freezing drizzle are extremely dangerous because they could freeze on the surface and make it rough. This will further reduce the laminar airflow and increase turbulent airflow [3]. When various components of the aircraft, such as the propeller or engine, are damaged or broken due to presence of ice, flight becomes impossible.

To solve this problem, anti-icing and de-icing chemicals can be applied to prevent or remove the formed ice if the freezing rain or drizzle could not get rid of the aircraft immediately. However, various anti-icing and deicing chemicals were reported that they contain urea, betaine or potassium formate, which could corrode the flight-critical components. Especially, when an aircraft was exposed to chemical tests, corrosion effects and weight losses were higher for urea than for the other chemicals [4]. On the other hand, the application of these chemicals could pose detrimental impacts to the environment and presents health risks to humans, when excessive chemicals were used where larger amount of heavy minerals were contained, or degradation happened [5]. Eventually, the dangers would be exacerbated. Benzotriazole is commonly used in anti-icing and de-icing agents, but only limited concentrations are permissible due to its toxicity (in water, $0.1 \mathrm{mg} / \mathrm{L}$; in air, $10 \mathrm{mg} / \mathrm{m}^{3}$ ) [6]. Glycol-based anti-icing agents are also used to de-ice planes, but they are toxic when ingested, and consume significant amounts of oxygen when released into the environment. This oxygen consumption depletes the dissolved oxygen in water which is harmful to aquatic life and could cause mass fish kills [7]. Agencies have been seeking for different alternatives to maximum the benefit while to minimize the drawbacks, such as anti-icer 1, 2propanediol. It is quite effective but very expensive, and it includes complicated procedures and byproducts with potential environmental concern during industry produc- 
tion [5]. Thus, there is an urgent need to find an effective and applicable method to approach this problem for aeronautic applications [8].

Recently, to an achieve an anti-icing surface, researchers turn to study of creation of superhydrophobic (SH) surfaces with nanostructures [9], nanoparticles [10] or anti-icing coatings $[4,11]$. A hydrophobic surface refers to a surface which yields a contact angle over $90^{\circ}$ for water, while a SH surface has a contact angle greater than $150^{\circ}$ with a low roll-off angle [12-16]. The mechanism of producing $\mathrm{SH}$ surface is to reduce the adhesion force between water/ice and the aircraft surface, and increase the probability that water will be driven away before the ice forms and starts accumulating [11]. Inspired by lotus leaf, Jiang et al. [17] designed a surface composed of micro ratchets and nano hairs on a metal substrate, and it was found that this surface had a very robust icephobic/anti-icing property better than both the single nanostructured microstructured surfaces by forming the Cassie-Baxter states. Gao et al. [10] created a composite SH surface by mixing organosilane-modified silica particles and polymer binder on an aluminum plate. The surfaces showed good anti-icing capability, which depends on the nano particle size; however, the critical size for icephobic property was significantly different from that of superhydrophobicity, and no further correlation was reported. The application of anti-icing chemicals, such as organosilicate coating (poly-dimethylphenylsiloxanes and polydimethylsiloxane- $\alpha, \omega$-diol), is a traditional method to prevent the formation of ice on the surface of an aircraft, and the objective is to decrease the intermolecular force between water/ice and the surface of a plane [11].

To further understand of the wetting on an anti-icing and de-icing surface, we would introduce the wetting behaviors of liquid on both smooth and rough solid surfaces first. On a perfectly smooth surface, Young's equation can be used to calculate the Young contact angle of a liquid with the interfacial surface tensions between solid, liquid, and vapor as follows $[18,19]$ :

$$
\cos \theta_{e}=\frac{\gamma_{S V}-\gamma_{S L}}{\gamma_{L V}}
$$

where $\theta_{e}$ is the Young contact angle, $\gamma_{S V}$ is the solid-vapor surface tension, $\gamma_{S L}$ is the solid-liquid surface tension, and $\gamma_{L V}$ is the liquid-vapor surface tension.

While on a rough surface, there are two predominant models to describe the wetting behaviors: Wenzel model and the Cassie-Baxter model. When a liquid completely wets a rough surface, the Wenzel equation relates to the roughness of the surface with the apparent contact angle as $[20,21]$ and it is called Wenzel state:

$$
\cos \theta_{r}^{w}=r \cos \theta_{e}
$$

where $\theta_{r}^{W}$ is the apparent contact angle in the Wenzel state, $r$ is the roughness of a surface which can be obtained from dividing the actual area by the projected area, and $\theta_{e}$ is the Young contact angle in Equation (1).

For Cassie-Baxter model, a liquid partially wets a rough surface, where an apparent contact angle is determined by [22]:

$$
\cos \theta_{r}^{C B}=f_{1} \cos \theta_{e}-f_{2}
$$

where $\theta_{r}^{C B}$ is the apparent contact angle in the CassieBaxter state, $f_{1}$ is the area of the liquid in contact with the solid divided by the projected area, and $f_{2}$ is the area of the liquid in contact with the air divided by the projected area. Marmur rewrote the Cassie-Baxter equation as [23]:

$$
\cos \theta_{r}^{C B}=r_{f} f \cos \theta_{e}+f-1
$$

where $r_{f}$ is the roughness of the total wetted area and $f$ is the fraction of the projected area of the liquid in contact with the solid surface. According to Equation (4), the contributions of surface roughness and trapped air are apparent when preparing a hydrophobic surface.

Given the above, even lots of study have been done on introducing superhydrophobicity to icephobicity, few researchers analyze the underlying mechanism of adhesion between two surfaces and there are few studies about generating anti-icing surfaces with only hydrophobic property. In this work, a hydrophobic aluminum surface was achieved by roughening it to make some grooves in micro-scale, followed by modifying the whole surface with fluorochemicals to decrease its surface tension [24, 25]. For this hydrophobic surfaces, air gaps would be formed amongst the grooves and protuberances on top of the surfaces when water sits on the surface (Cassie-Baxter state), which renders it to be a liquid-air-solid composite structure beneath the liquid. Meanwhile, compared with a smooth surface, the adhesion force between water/ ice and solid would be decreased significantly due to the air gaps. Therefore, with this hydrophobic and low-adhesion aluminum surface, the anti-icing and de-icing properties can be achieved, and the cost will decreased, which has potential to be applied to aircraft industry.

\section{Experimental}

\subsection{Materials}

Aluminum panels (from Air Force Research Laboratory) were used as smooth as well as rough surfaces. Heptadecafluoro-1,1,2,2-tetrahydrodecyltrimethoxysilane (fluorosilane, $\mathrm{C}_{13} \mathrm{H}_{13} \mathrm{~F}_{17} \mathrm{O}_{3} \mathrm{Si}$, Gelest), tetramethylorthosilicate (TMOS, $\mathrm{C}_{4} \mathrm{H}_{12} \mathrm{O}_{4} \mathrm{Si}$, Aldrich), hydrogen chloride $(\mathrm{HCl}$, Aldrich), ammonium hydroxide $\left(\mathrm{NH}_{4} \mathrm{OH}\right.$, Mallinckrodt Chemical), acetone $\left(\mathrm{C}_{3} \mathrm{H}_{6} \mathrm{O}\right.$, Aldrich), isopropyl alcohol $\left(\mathrm{C}_{3} \mathrm{H}_{7} \mathrm{OH}\right.$, Fisher), and distilled water were used without further purification. 


\subsection{Grafting of Fluorosilane on a Smooth Aluminum Surface}

Surface tension of the aluminum surface can be reduced by grafting fluorosilane onto its surface. A solution of $2 \%$ TMOS, $4 \%$ fluorosilane, and $10 \% 0.1 \mathrm{M} \mathrm{HCl}$ solution were mixed in isopropyl alcohol and allowed to sit with intermittent vortexing for 20 - 30 minutes, until the siloxanes were hydrolyzed. The aluminum panel was laid on a hot plate set to $60^{\circ} \mathrm{C}-80^{\circ} \mathrm{C}$ to encourage oxidization and increase $\mathrm{Al}_{2} \mathrm{O}_{3}$ formation on the aluminum surface. A piece of cotton fabric was dipped into the prepared solution, and the hot panels were rubbed with the cotton fabric. The panels were rinsed with isopropyl alcohol and dried in air to remove the residue.

\subsection{Grafting of Fluorosilane on a Rough Aluminum Surface}

An aluminum panel was roughened using sand papers with the combination of reducing the surface energy via chemical modification to achieve a Cassie-Baxter state for water. The roughness of the panel should be appropriate, since an overly roughened aluminum surface used for aircraft exterior can increase drag during take-off and result in unbalanced flying, while insufficient roughness would increase the contact area between water and aluminum panel and make it difficult get rid of the water. Study showed that roughness in micro-scale was effective on an aluminum panel; thus the panel was roughened with 600 grit sand papers, followed by being washed with water and isopropyl alcohol. The rough aluminum panel was treated with fluorosilane just as the smooth panels. During the process of surface modification, all the conditions must be kept exactly the same for smooth and rough aluminum panels.

\subsection{Characterization}

\subsubsection{Scanning Electron Microscopy}

The fluorosilane-treated aluminum panel and control sample were observed with a scanning electron microscope (SEM, JEOL, 6400F, Tokyo, Japan) operated at 10 $\mathrm{kV}$ and magnifications from $\times 500$. Revolution ${ }^{\mathrm{TM}}(4 \mathrm{pi}$ Analysis, v1.60b24) were used for image analysis of the SEM images.

\subsubsection{Water Contact Angles}

The apparent contact angles of water drops on the surfaces of the prepared materials and the controls were measured with a lab-designed goniometer at room temperature (RT) and $65 \%$ relative humidity $(\mathrm{RH})$. The volume of the deposited droplets was $10 \mu \mathrm{L}$. The range of contact angles was obtained from measurements taken at a minimum of three separate locations. The images of liquid droplets on the prepared surface were taken using a digital camera (Canon, EOS EF-S-18-55IS, Lake Success, NY, USA) that has an optical microscopic focusing lense (Meiji Techno, EMZ-13TR, Saitama, Japan).

\subsubsection{Anti-Icing Properties}

Fluorosilane-treated and untreated aluminum panels were placed in a freezer at an average temperature of $-8^{\circ} \mathrm{C}$ and $57 \% \mathrm{RH}$ for 13 hours. A bottle of distilled water was kept in the freezer at an average temperature of $-8^{\circ} \mathrm{C}$ for 13 hours while ensuring that it did not freeze. The distilled water below its freezing point would not crystallize or freeze if it is lack of a seed crystal or nucleus, and it is called "super-cooled water". Immediately after removing both the water and the panel from the freezer, the panels were placed on a platform inclined at $10^{\circ}$, and supercooled water was poured onto the surfaces at RT and $55 \% \mathrm{RH}$.

\subsubsection{De-Icing Properties}

A $200 \mu \mathrm{L}$ distilled water drop was deposited onto both the fluorosilane-treated and the untreated aluminum panels. The panels along with water drops were carefully placed in a freezer and maintained at an average temperature of $-13^{\circ} \mathrm{C}$ and $51 \% \mathrm{RH}$ for 12 hours in order to completely freeze the water drops. The samples with ice crystals were removed from the freezer and laid on an inclined platform where the tilt angle was $45^{\circ}$. Photographs of the melting process were taken at RT with a digital camera.

\section{Results and Discussions}

To achieve anti-icing and de-icing properties from a hydrophobic surface, a Cassie-Baxter state should be formed when water drops are deposited on this surface. Both geometrical and chemical modifications can help to improve the wetting behavior of a solid material to form a Cassie-Baxter state. Increasing the surface roughness leads to more air gaps between protuberances (geometrically), while decreasing surface tension results in the increase of the Young contact angle (chemically). Therefore, roughening an aluminum surface followed by coating with a low-surface-tension chemical, such as fluorosilane, will make the surface more hydrophobic compared with a smooth untreated surface. Also, it can improve the anti-icing and de-icing properties of the aluminum surface due to the decrease of adhesion.

\subsection{Chemical Modification}

Fluorosilane $\left(\gamma_{L V}=12 \mathrm{mN} / \mathrm{m}\right)$ has been grafted on both smooth and roughened aluminum surfaces to decrease its surface energy as shown in Scheme 1, where - $\mathrm{R}$ refers to $-\left(\mathrm{CH}_{2}\right)_{2}\left(\mathrm{CF}_{2}\right)_{5} \mathrm{CF}_{3}$. Fluorosilane and TMOS were first 
hydrolyzed and formed crosslink in the solution, and then reacted with the hydroxyl groups attracted to aluminum oxide surface due to the oxidation of aluminum. Thus, a thin network would be obtained and it leads to the hy- drophobicity. As shown in Figure 1, the hydrolyzed fluorosilane on the rough aluminum surface slightly increased the roughness at nano-scale.

Fluorosilane-treated on aluminum not only increased

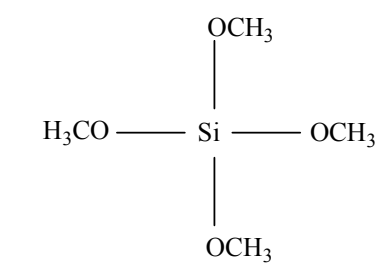

TMOS

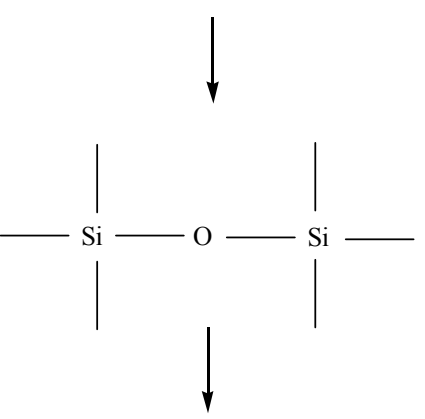



Fluorosilane<smiles>C1CCCCC1</smiles>

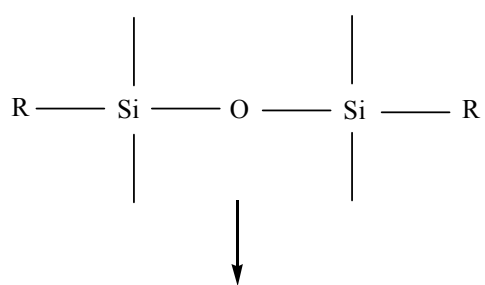

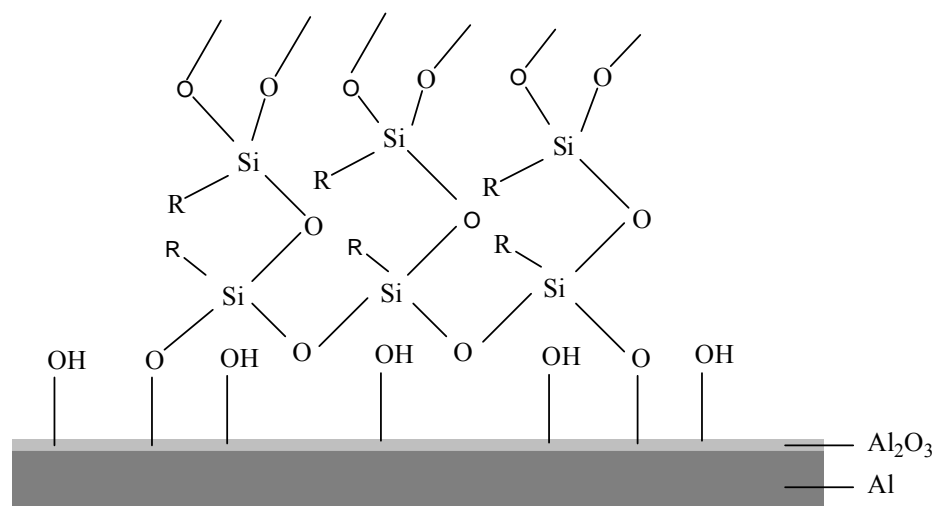

Scheme 1. Surface modification of the aluminum panel with fluoro chemicals.

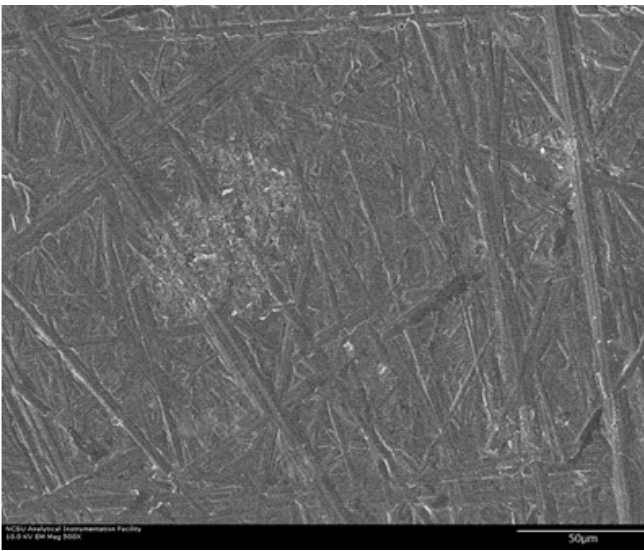

(a)

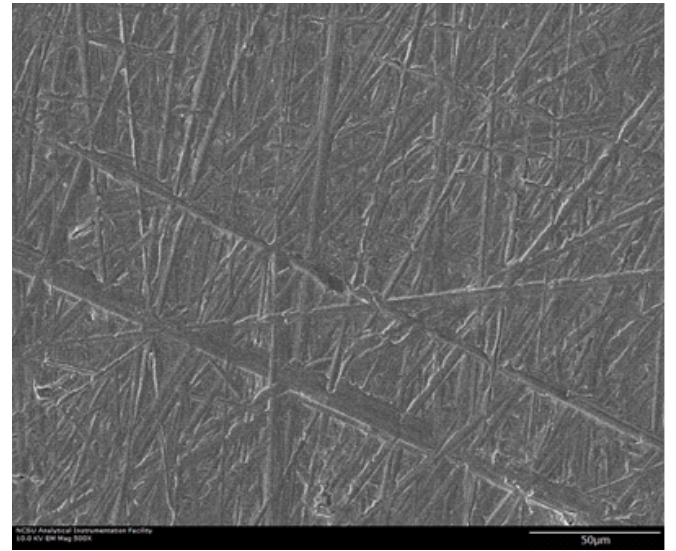

(b)

Figure 1. SEM image of rough aluminum surfaces: (a) fluorosilane-treated $(\times 500)$ and $(b)$ untreated $(\times 500)$. 
nano-scale roughness but also reduced the surface tension of the aluminum. This has been verified by compareing the contact angles of water on treated and untreated smooth surfaces. The average Young contact angle, $\theta_{e}$, of water on an untreated smooth surface is $(62 \pm 2)^{\circ}$, while on the fluorosilane-treated surface is $(112 \pm 2)^{\circ}$ as shown in Figure 2. This indicates that fluorosilane altered the hydrophilic aluminum surface to be a hydrophobic surface, which can be more hydrophobic if the surface is more roughened.

\subsection{Geometrical Modification}

By increasing $r$ in Equation (2), or reducing $f_{1}$ and increasing $f_{2}$ in Equation (3), a hydrophobic surface can become more hydrophobic. As shown in Figure 1, the sanded rough surface has a number of narrow grooves and wide protuberances. If there are air pockets between solid protuberances on a rough surface, Cassie-Baxter model can be used to predict the apparent contact angle. As shown in Figure 3, by modifying Equation (3), the apparent contact angle on this Cassie-Baxter surface is obtained by [24]:

$$
\cos \theta_{r}^{C B}=\frac{R\left(\pi-\theta_{e}\right)+A}{R+D+A} \cos \theta_{e}+\frac{R \sin \theta_{e}+A}{R+D+A}-1
$$

where $R$ is the radius of the curvy part of the grooves, $2 D$

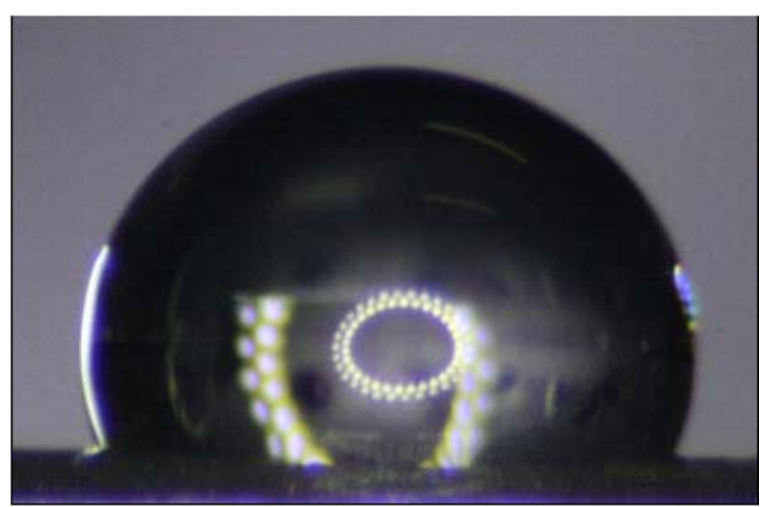

(a)

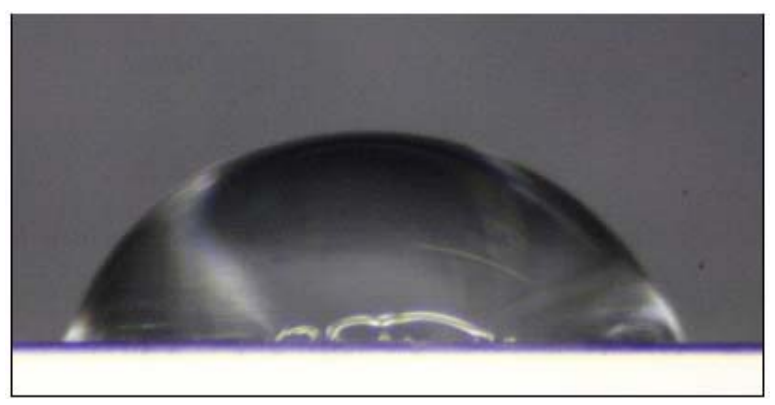

(b)

Figure 2. A $10 \mu \mathrm{L}$ water droplet on (a) a fluorosilanetreated smooth aluminum panel and (b) an untreated smooth aluminum panel.

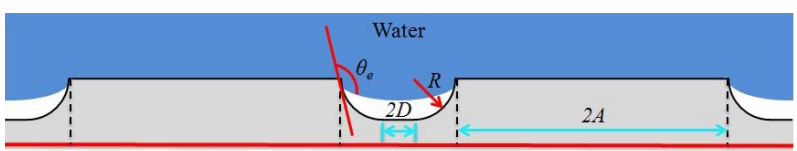

Figure 3. A water drop sitting on top of the fluorosilanetreated aluminum surface: a Cassie-Baxter rough surface.

is the edge-to-edge distance between two protuberances, and $2 A$ is the width of the flat top protuberances.

For structure of the aluminum panels, the distance between protuberances should be designed properly to achieve the Cassie-Baxter state. A structure with extremely large $2 D$ will lower robustness due to the increase of the sagging height at the liquid-vapor interface, and this will eventually result in a Wenzel model. Meanwhile, a structure with extremely small $2 D$ is not able to generate enough air pockets underneath the liquid, and this will prevent the surface from being more hydrophobic. As mentioned, $R$ is very small compared to the $2 D$ and $2 A$, and is negligible. Since $R \approx 0$, Equation (5) can be rewritten as:

$$
\cos \theta_{r}^{C B}=\frac{A}{D+A} \cos \theta_{e}+\frac{A}{D+A}-1
$$

On the fluorosilane-treated aluminum rough surfaces, the average $A$ and $D$ are approximately $(7.21 \pm 1.00) \mu \mathrm{m}$ and $(1.26 \pm 0.25) \mu \mathrm{m}$, respectively, while $\theta_{e}=(112 \pm 2)^{\circ}$ as shown in Figure 2(a). By substituting these parameters into Equation (6), the average Cassie-Baxter apparent contact angle of water on this fluorosilane-treated rough surface is obtained, $\theta_{r}^{C B}=117.9$ while the measured apparent contact angle is approximately $(118.0 \pm 2)^{\circ}$ as shown in Figure 4(a). For an untreated rough surface, the hydrophilic surface will become more hydrophilic in a Wenzel state. In Figure 4(b), the apparent contact angle is $(60 \pm 2)^{\circ}$, slightly smaller than that in Figure 2(a) which is $(62 \pm 2)^{\circ}$. Therefore, the predicted value obtained from our modeling shows a good agreement with the experimental results.

In addition, the roll-off properties of the fluorosilanetreated and untreated rough aluminum panels were evaluated. Previous research [26] discussed the relationship among the roll-off angle, droplet size, mass, advancing and receding contact angles on a rough surface as:

$$
m g \sin \alpha / l=\gamma_{L V}\left(\cos \theta_{R}-\cos \theta_{A}\right)
$$

where $m$ is the mass of the drop, $g$ is gravitational acceleration, $\alpha$ is the roll-off angle, $l$ is the length of the drop on a tilting surface, $\theta_{R}$ is receding contact angle, and $\theta_{A}$ is advancing contact angle.

The roll-off angle of a droplet on a solid surface depends on density and volume of the liquid. Large drops easily roll off at a small tilt angle, while small drops roll off at a large tilt angle, and it even does not roll off. In 


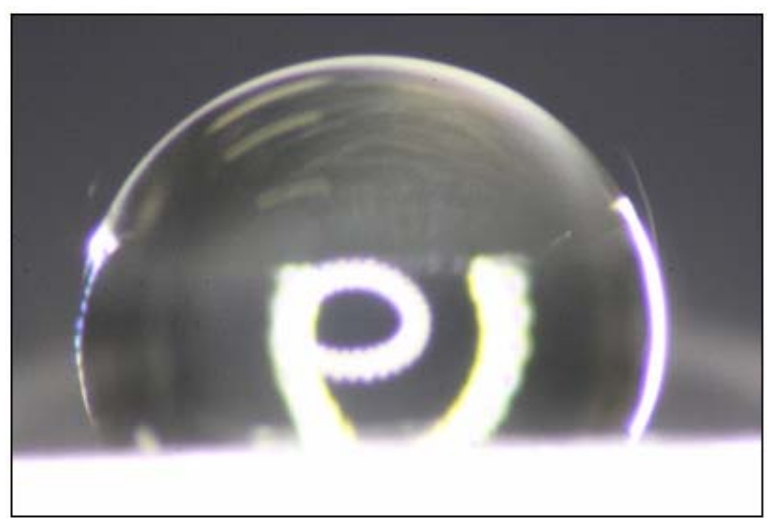

(a)

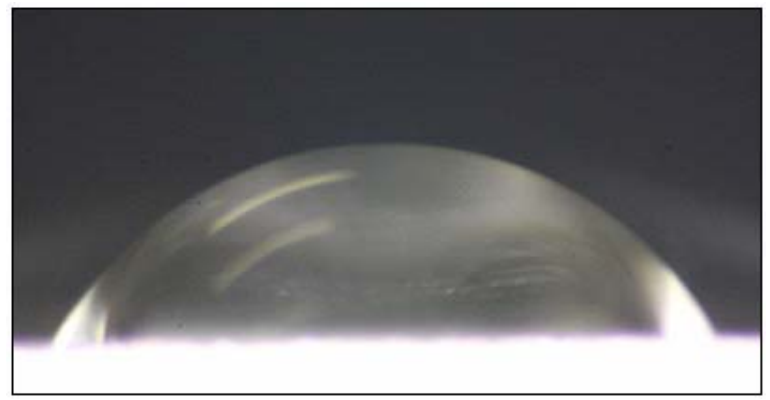

(b)

Figure 4. A $10 \mu \mathrm{L}$ water droplet on a rough aluminum surfaces: (a) fluorosilane-treated and (b) untreated.

this experiment, a volume of $50 \mu \mathrm{L}$ water was deposited on solid surfaces. On the fluorosilane-treated and untreated aluminum panels, the $l$ are $(2.39 \pm 0.20)$ and $(4.81$ $\pm 0.20) \mathrm{mm}$, the $\theta_{R}$ are $(69 \pm 2)^{\circ}$ and $(18 \pm 2)^{\circ}$, and the $\theta_{A}$ are $(123 \pm 2)^{\circ}$ and $(106.0 \pm 2)^{\circ}$, respectively. When these parameters are substituted into Equation (7), the predicted roll-off angles are obtained: $\alpha=32.7^{\circ}$ for the treated aluminum, while $\alpha=64.4^{\circ}$ for the untreated one. As shown in Figure 5, the measured roll-off angles for the treated and untreated panels are $(35.0 \pm 2)^{\circ}$ and $(64.0$ $\pm 2)^{\circ}$, respectively. The predicted and measured roll-off angles show good agreement within a small error, and this property can be extended to the evaluation of the anti-icing and de-icing properties of the treated rough surfaces.

\subsection{Anti-Icing Properties of Fluorosilane Treated Aluminum Panel}

The anti-icing properties were tested by pouring supercooled water onto a $10^{\circ}$ inclined fluorosilane-treated and untreated rough aluminum surfaces.

As shown in Figure 6, the super-cooled water formed ice immediately on the untreated aluminum surface and accumulated gradually, while it just slid down the treated panel without nucleating or forming any ice on it. The different wetting behaviors between treated and untreated

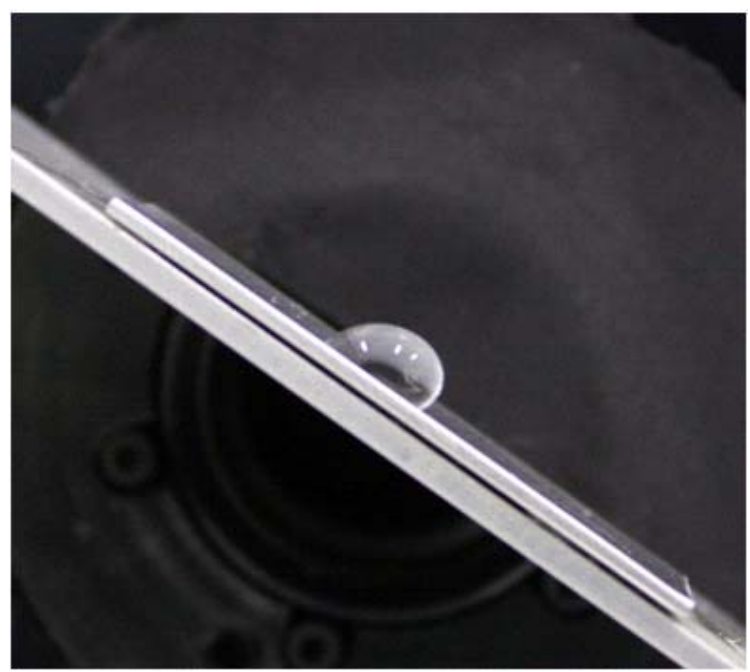

(a)

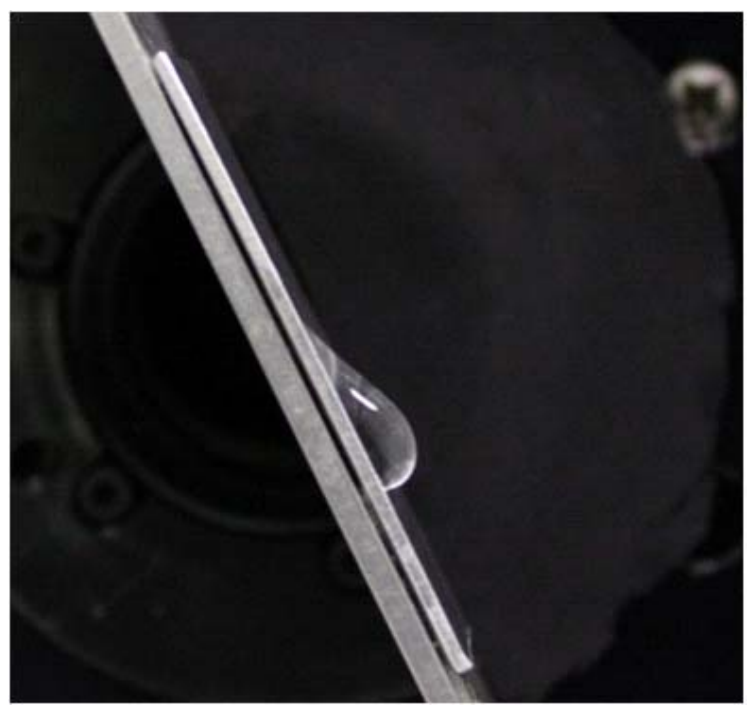

(b)

Figure 5. Roll-off angles of $50 \mu \mathrm{L}$ water on aluminum panels: (a) fluorosilane-treated $\left(35^{\circ}\right)$ and (b) untreated $\left(64^{\circ}\right)$.

rough surfaces imply that the hydrophobic rough surface, which is a Cassie-Baxter surface, can provide sufficient anti-icing properties by generating air gaps among the protuberances, while the hydrophilic surface has large water-ice-solid contact area and high surface tension, which leads to a large adhesion force. Thus, the untreated aluminum panel cannot prevent the super-cooled water from rolling off the surface.

The two different behaviors largely depend on the adhesion forces between the two surfaces when supercooled water is deposited on the aluminum surfaces [1,27-31]. Adhesion force refers to the interactions between different molecules, and in this work, it is between the molecules in water/ice and the molecules in aluminum panel. As shown in Figure 7, four different models 


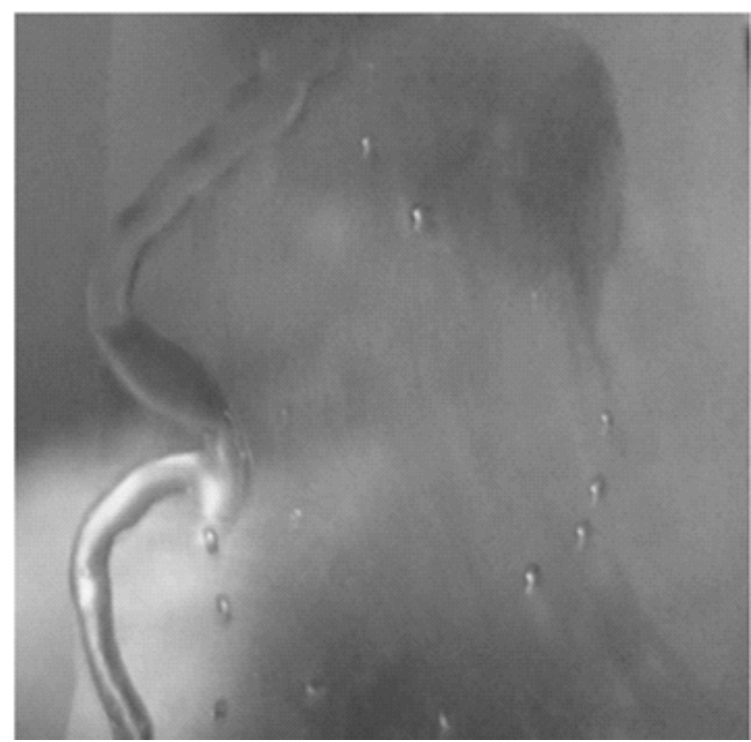

(a)

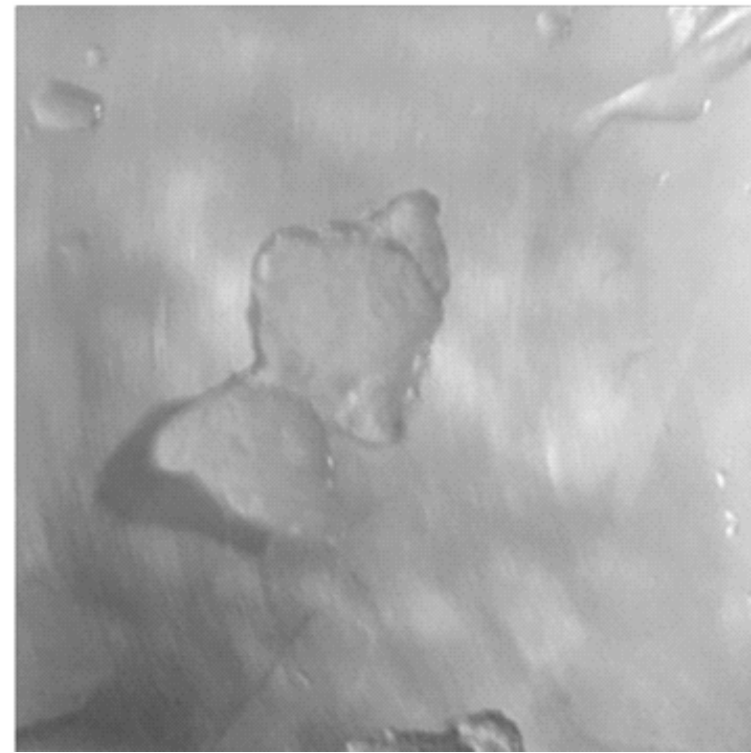

(b)

Figure 6. Super-cooled water (a) running off on a fluorosilane-treated aluminum surface and (b) building ice on an untreated aluminum surface.

are designed to discuss the effects of adhesion forces on anti-icing properties. In Figure 7(a), a small sessile water drop sits on a perfectly smooth surface in thermodynamic equilibrium. In horizontal direction, Equation (1) depicts the relationships between three interfacial energies; in perpendicular direction, the adhesion force balances the partial liquid-vapor interfacial energy if gravity is negligible:

$$
F_{a}=\pi r_{a}^{2} P_{a}-\gamma_{L V} \sin \theta_{e} \cdot 2 \pi r_{a}
$$

where $F_{a}$ refers to the adhesion force for the case in Figure 7(a), $r_{a}$ refers to the radius of liquid-solid contact

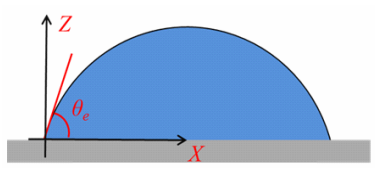

(a)



(c)

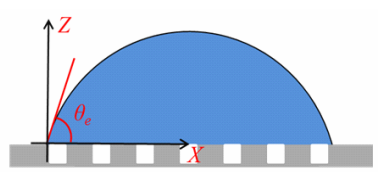

(b)

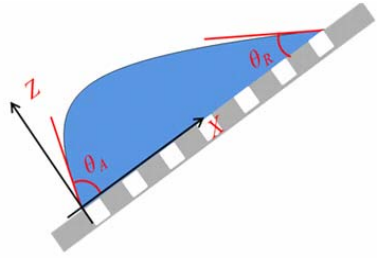

(d)
Figure 7. Adhesion forces between water and aluminum surfaces for different wetting states. (a) A sessile water drop sits on a flat and perfectly smooth aluminum surface; (b) a sessile water drop sits on a flat but rough aluminum surface; (c) a water drop sits on a tilt and smooth aluminum surface; and (d) a water drop sits on a tilt but rough aluminum surface.

area, and $P_{a}$ refers to the Laplace pressure. When the solid surface has been changed into a rough surface, the contact angle and liquid-solid contact area will change accordingly. The adhesion force will be:

$$
F_{b}=\pi r_{b}^{2} P_{b}-\gamma_{L V} \sin \theta_{r}^{C B} \cdot 2 \pi r_{b} \lambda
$$

where $F_{b}$ refers to the adhesion force for the case in Figure 7(b), $r_{b}$ refers to the radius of liquid-solid contact area, $P_{b}$ refers to the Laplace pressure, and $\lambda$ refers to corrective coefficient since the liquid-vapor-solid contact line is discontinuous on a rough surface $(0<\lambda<1)$. Combining Equation (9) with Equation (4), the forces can be obtained both for Wenzel and Cassie-Baxter states. Under the same conditions, for a liquid with infinite volume, the adhesion force is always smaller on a smooth surface than that of a liquid on a rough surface in Wenzel state, while it is larger than the force when it is in Cassie-Baxter state, due to the change of liquid-solid contact areas. While for a liquid with fixed volume on a rough surface, it depends on the dominant component. When Laplace pressure and the change of liquid-solid contact area are dominant, similar conclusion can be made with the infinite volume; when the change of apparent contact angle and contact line length are dominant, the adhesion force will increase if the surface is hydrophilic, or decrease if the surface is hydrophobic. Above all, the adhesion force between water/ice and the aluminum will become smaller if a Cassie-Baxter state exists.

When a drop is deposited on a tilt the surface, the adhesion force will be different as shown in Figures 7(c) and $(\mathbf{d})$. There are the advancing $\left(\theta_{A}\right)$ and receding $\left(\theta_{R}\right)$ contact angles at the two ends of the drops, which indicates at each point of the liquid-vapor-solid contact line, the contact angle will be different and in a range of $\theta_{R}$ to 
$\theta_{A}$. Therefore, in case (C), if the liquid-vapor-solid contact line is divided into $N$ segments, the total adhesion force will be:

$$
F_{c}=\pi r_{c}^{2} P_{c}-\sum_{i=1}^{N-1} \gamma_{L V} \sin \left(\theta_{R}+\frac{\theta_{A}-\theta_{R}}{N-1} \cdot i\right) \cdot \frac{2 \pi r_{c}}{N}
$$

where $F_{c}$ refers to the adhesion force for the case in Figure 7(c), $r_{c}$ refers to the radius of liquid-solid contact area since an assumption is made that the contact area is a circle, and $P_{c}$ refers to the Laplace pressure. While on a rough surface, the adhesion force can be achieved similarly by introducing the corrective coefficient:

$$
F_{d}=\pi r_{d}^{2} P_{d}-\sum_{i=1}^{N-1} \gamma_{L V} \sin \left(\theta_{R}+\frac{\theta_{A}-\theta_{R}}{N-1} \cdot i\right) \cdot \frac{2 \pi r_{d}}{N} \cdot \lambda
$$

where $F_{c}$ refers to the adhesion force for the case in Figure 7(d), $r_{c}$ refers to the radius of liquid-solid contact area, $P_{c}$ refers to the Laplace pressure, and $\lambda$ refers to corrective coefficient since the liquid-vapor-solid contact line is discontinuous on a rough surface $(0<\lambda<1)$. Comparing Equations (10) and (11), the contact angle hysteresis $\left(\Delta \theta=\theta_{A}-\theta_{R}\right)$, the radius of liquid-vapor-solid contact area and Laplace pressure have a major impact on the adhesion force. In our study, the surface is designed by increasing the roughness and decreasing the surface tension, which can increase the static contact angle and contribute a lot to the decrease of the adhesion force. For the untreated and treated rough aluminum surface, the contact angle hystereses are $(98 \pm 2)^{\circ}$ and $(44 \pm$ $2)^{\circ}$, and the contact line lengths are $(4.81 \pm 0.20) \mathrm{mm}$ and $(2.39 \pm 0.20) \mathrm{mm}$. When $N=20$ and $\lambda=0.85$, it is observed that that the adhesion forces due to surface tension for untreated and treated surfaces are $1.70 \mathrm{mN}$ and 0.84 $\mathrm{mN}$; the adhesion forces due to Laplace pressure are not straightforward but they should be approximately the same, since on the flat surfaces, the Laplace pressure for the untreated and treated surfaces are quite close, which are $1.46 \mathrm{mN}$ and $1.71 \mathrm{mN}$, respectively. Therefore, approximately we can obtain $F_{c}>F_{d}$. It is apparent that the adhesion force has been decreased significantly, which implies that for the same liquid, weak adhesion leads to poor wetting and good anti-icing property.

\subsection{De-Icing Properties of Fluorosilane Treated Aluminum Panel}

The de-icing properties of the fluorosilane-treated and untreated rough aluminum surfaces were also evaluated as shown in Figure 8. A $200 \mu \mathrm{L}$ distilled water drop was deposited on each treated and untreated aluminum panel, and the panels with water were stored in a freezer for 12 hours. As soon as panels were moved to a $45^{\circ}$ inclined mount, the melting process had been observed to evaluate the de-icing properties of the surfaces. The droplet


(a)
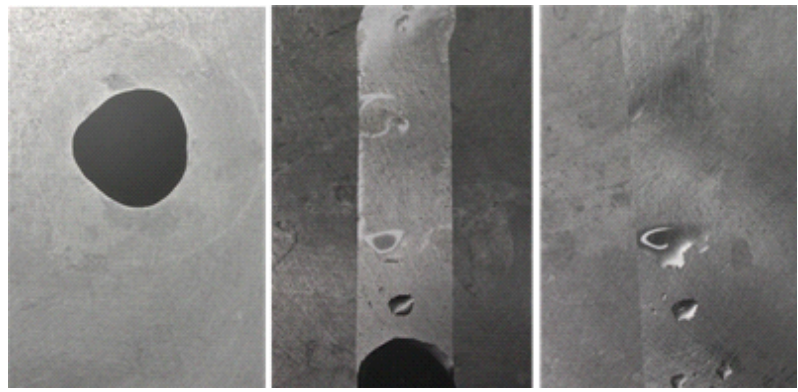

(b)

Figure 8. Deicing properties of (a) fluorosilane-treated aluminum panel and (b) untreated aluminum panel at $45^{\circ}$ tilt angle. While ice cleanly melt and slide down from the treated surface, ice slide down leaving trails behind while sliding down.

rolled off from the fluorosilane-treated aluminum surface after 35 seconds, while on the untreated surface the water drop melted at the same rate but could not roll off. It took 43 seconds for the drop to reach the edge of the untreated panel, but the drop stayed at the edge for 7 seconds before sliding off. In addition, there was almost no residue on the treated surface after water rolled off, but water left its trail while sliding off the untreated panel. This is caused by the difference of $f_{1}, f_{2}$, and the apparent contact angles between two panels. According to our models, the treated rough surface has less $f_{1}$, more $f_{2}$, a larger apparent contact angle, and less direct contact with water than the untreated one has. To quickly remove the ice from the aluminum surfaces, thermal energy is required to melt the ice where directly contacts with the aluminum surface. Therefore, a larger contact area will require more energy to melt the ice and completely remove water from the aluminum surface. This can be explained by the differential form of Fourier's law of thermal conduction, $q=-k A(d T / d x)$, where $q$ is heat flow, $k$ is thermal conductivity, $A$ is the total area of the material, $d T$ is the temperature deference between two ends, and $d x$ is the distance between two end. According to Fourier's law, when the aluminum panel with ice is taken out from the freezer and exposed to the room temperature (RT), the heat energy in the air rarely contributes to the de-icing process even if the $A$ of ice is equal to the $A$ of aluminum 
since the $k$ of air, $0.025 \mathrm{~W} / \mathrm{m} \cdot \mathrm{K}$, is significantly smaller, if not negligible, than that of aluminum, $205 \mathrm{~W} / \mathrm{m} \cdot \mathrm{K}$, while the $k$ of water is $0.596 \mathrm{~W} / \mathrm{m} \cdot \mathrm{K}$. For the treated aluminum, $10.7 \mathrm{~kJ}$ is required to make the ice slide off; while for the untreated aluminum, $25.7 \mathrm{~kJ}$ is needed to melt the ice contacting with the panel. In addition, the amount of thermal energy required to change the temperature of a material, specific heat capacity, of water is approximately four times greater than that of aluminum, e.g. $c_{p}($ water $)=4.181 \mathrm{~J} / \mathrm{g} \cdot \mathrm{K}$ while $c_{p}($ aluminum $)=0.897$ $\mathrm{J} / \mathrm{g} \cdot \mathrm{K}$ at RT. Meanwhile, Fourier's law of thermal conduction is a form integrated by time, and therefore the temperature of aluminum changes faster than ice does when both materials are exposed to the same thermal energy. Once the temperature of aluminum reaches RT, the total $q$ required for de-icing largely depends on the $A$, i.e. the lower $f_{1}$ in the Cassie-Baxter model the lower $q$ for de-icing. It is clear that a Cassie-Baxter surface have better anti-icing and de-icing properties than a smooth surface or a Wenzel surface does.

\section{Conclusions}

An anti-icing and de-icing aluminum surface was designed and developed by modifying the physical topography and decreasing its surface energy. Grafting fluorosilane onto the smooth aluminum surface altered the wetting behavior of the material, and the hydrophilic surface became hydrophobic. By adding micro-scale roughness to the fluorosilane-treated surface, the apparent contact angle increased up to $118^{\circ}$ and became a Cassie-Baxter surface; by adding the same roughness to the untreated surface, the contact angle decreased and became a Wenzel surface. In addition, a water drop on a fluorosilanetreated surface can roll off at a significantly smaller rolloff angle than that deposited on an untreated surface due to the different $f_{1}$ and $f_{2}$ between modified and unmodified surfaces.

The differences in anti-icing properties of the fluorosilane-treated aluminum rough surface and the control are obvious when super-cooled water was poured onto the surfaces. In the process, ice built up on the untreated rough surface, but the super-cooled water completely slid off leaving no residue behind on the treated rough surface. The de-icing properties of the same materials have been evaluated as well. The ice on the treated surface can be removed easily from the surface while the ice built up on the untreated surface leaves a significant amount of trail behind after barely sliding off. Therefore, it is verified that the chemical and physical modifications of a solid surface can improve its anti-icing and de-icing properties with only hydrophobic property, which might be applied to the modification of aircraft exterior to prevent the formation of ice and to assist with the melting of ice on the aluminum surface. Meanwhile, the cost will be reduced compared with the creation of $\mathrm{SH}$ surface to achieve an anti-icing and de-icing surface.

\section{REFERENCES}

[1] C. Laforte and A. Beisswenger, "Icephobic Material Centrifuge Adhesion Test," Proceedings of the International Workshop on Atmospheric Icing of Structures (IWAIS XI), Montréal, 16 June 2005, pp. 1-5.

[2] F. T. Lynch and A. Khodadoust, "Effects of Ice Accretions on aircraft Aerodynamics," Progress in Aerospace Sciences, Vol. 37, 2001, pp. 669-767.

[3] B. C. Bernstein, T. A. Omeron, M. K. Politovich and F. McDonough, "Surface Weather Features Associated with Freezing Precipitation and Severe In-Flight Aircraft Icing," Atmospheric Research, Vol. 46, No. 1-2, 1998, pp. 57-73. doi:10.1016/S0169-8095(97)00051-3

[4] E. Huttunen-Saarivirta, V.-T. Kuokkala, J. Kokkonen and H. Paajanen, "Corrosion Effects of Runway De-Icing Chemicals on Aircraft Alloys and Coatings," Materials Chemistry and Physics, Vol. 126, No. 1-2, 2011, pp. 138151. doi:10.1016/j.matchemphys.2010.11.049

[5] L. Fay and X. Shi, "Environmental Impacts of Chemicals for Snow and Ice Control: State of the Knowledge," Water, Air, \& Soil Pollution, Vol. 223, No. 5, 2012, pp. 2751-2770. doi:10.1007/s11270-011-1064-6

[6] D. E. A. C. Ancilla, A. N. K. E. H. Oltkamp, L. U. C. A. M. Atassa and X. I. F. Ang, "Isolation and Characterization of Microtox-Active Components from Aircraft DeIcing/Anti-Icing Fluids," Environmental Toxicology and Chemistry, Vol. 16, No. 3, 1997, pp. 430-434. doi:10.1002/etc. 5620160306

[7] R. A. Kent, D. Andersen, P. Caux and S. Teed, "Canadian Water Quality Guidelines for Glycols: An Ecotoxicological Review of Glycols and Associated Aircraft Anti-Icing and Deicing Fluids," Environmental Toxicology, Vol. 14, No. 5, 1999, pp. 481-522.

doi:10.1002/(SICI)1522-7278(199912)14:5<481::AID-T OX5>3.0.CO;2-8

[8] A. Alizadeh, M. Yamada, R. Li, W. Shang, S. Otta, S. Zhong, L. Ge, A. Dhinojwala, K. R. Conway, V. Bahadur, A. J. Vinciquerra, B. Stephens and M. L. Blohm, "Dynamics of Ice Nucleation on Water Repellent Surfaces," Langmuir, Vol. 28, No. 6, 2012, pp. 3180-3186. doi:10.1021/1a2045256

[9] L. Mishchenko, B. Hatton, V. Bahadur, J. A. Taylor, T. Krupenkin and J. Aizenberg, "Design of Ice-Free Nanostructured Impacting Water Droplets," ACS NANO, Vol. 4 , No. 12, 2010, pp. 7699-7707. doi:10.1021/nn102557p

[10] L. Cao, A. K. Jones, V. K. Sikka, J. Wu and D. Gao, "Anti-Icing Superhydrophobic Coatings," Langmuir, Vol. 25, No. 21, 2009, pp. 12444-12448. doi:10.1021/la902882b

[11] S. V. Chuppina, "Anti-Icing Gradient Organosilicate Coatings," Glass Physics and Chemistry, Vol. 33, No. 5, 2007, pp. 502-509. doi:10.1134/S1087659607050136

[12] H. J. Lee and J. R. Owens, "Motion of Liquid Droplets on a Superhydrophobic Oleophobic Surface," Journal of Ma- 
terials Science, Vol. 46, No. 1, 2010, pp. 69-76. doi:10.1007/s10853-010-4810-Z

[13] H. J. Lee and J. R. Owens, "Design of Superhydrophobic Ultraoleophobic NyCo," Journal of Materials Science, Vol. 45, No. 12, 2010, pp. 3247-3253. doi:10.1007/s10853-010-4332-8

[14] H. J. Lee, "Design and DEvelopment of Anti-Icing Textile Surfaces," Journal of Materials Science, Vol. 47, No. 13, 2012, pp. 5114-5120. doi:10.1007/s10853-012-6386-2

[15] H. J. Lee and S. Michielsen, "Preparation of a Superhydrophobic Rough Surface," Journal of Polymer Science Part B: Polymer Physics, Vol. 45, No. 3, 2006, pp. 253261. doi:10.1002/polb.21036

[16] H. J. Lee, "Design of Artificial Lotus Leaves Using Nonwoven Fabric," Journal of Materials Science, Vol. 44, No. 17, 2009, pp. 4645-4652. doi:10.1007/s10853-009-3711-5

[17] P. Guo, Y. Zheng, M. Wen, C. Song, Y. Lin and L. Jiang, "Icephobic/Anti-Icing Properties of Micro/Nanostructured Surfaces," Advanced Materials, Vol. 24, No. 19, 2012 , pp. 2642-2648. doi:10.1002/adma.201104412

[18] T. Young, “An Essay on the Cohesion of Fluid," Philosophical Transactions of the Royal Society, Vol. 95, No. 1805, 1805, pp. 65-87.

[19] R. Tadmor, "Line Energy and the Relation between Advancing, Receding, and Young Contact Angles," Langmuir, Vol. 20, No. 18, 2004, pp. 7659-7664. doi:10.1021/la049410h

[20] R. N. Wenzel, "Resistance of Solid Surfaces to Wetting by Water," Industrial \& Engineering Chemistry, Vol. 28, No. 8, 1936, pp. 988-994. doi:10.1021/ie50320a024

[21] G. McHale, "Cassie and Wenzel: Were They Really So Wrong?" Langmuir, Vol. 23, No. 15, 2007, pp. 82008205. doi:10.1021/la7011167

[22] A. B. D. Cassie and S. Baxter, "Wettability of Porous Surfaces," Transactions of the Faraday Society, Vol. 5, No. 5, 1944, pp. 546-551. doi:10.1039/tf9444000546

[23] A. Marmur, "Wetting on Hydrophobic Rough Surfaces:
To Be Heterogeneous or Not To Be?" Langmuir, Vol. 1, No. 2, 2003, pp. 8343-8348. doi:10.1021/la0344682

[24] R. Saraf, H. J. Lee, S. Michielsen, J. Owens, C. Willis, C. Stone and E. Wilusz, "Comparison of Three Methods for Generating Superhydrophobic, Superoleophobic Nylon Nonwoven Surfaces," Journal of Materials Science, Vol. 46, No. 17, 2011, pp. 5751-5760. doi:10.1007/s10853-011-5530-8

[25] L. Barbieri, E. Wagner and P. Hoffmann, "Water Wetting Transition Parameters of Perfluorinated Substrates with Periodically Distributed Flat-Top Microscale Obstacles," Langmuir, Vol. 23, No. 4, 2007, pp. 1723-1734. doi:10.1021/1a0617964

[26] C. G. L. Furmidge, "Studies at Phase Interfaces," Journal of Colloid Science, Vol. 17, No. 4, 1962, pp. 309-324, doi:10.1016/0095-8522(62)90011-9

[27] S. A. Kulinich and M. Farzaneh, "Ice Adhesion on SuperHydrophobic Surfaces," Applied Surface Science, Vol. 255, No. 18,2009 , pp. 8153-8157. doi:10.1016/j.apsusc.2009.05.033

[28] D. K. Sarkar and M. Farzaneh, "Superhydrophobic Coatings with Reduced Ice Adhesion," Journal of Adhesion Science and Technology, Vol. 23, No. 9, 2009, pp. 12151237. doi:10.1163/156856109X433964

[29] W. D. Bascom, R. L. Cottington and C. R. Singleterry, "Ice Adhesion to Hydrophilic and Hydrophobic Surfaces," The Journal of Adhesion, Vol. 1, No. 1-4, 1969, pp. 246263. doi:10.1080/00218466908072188

[30] A. Dotan, H. Dodiuk, C. Laforte and S. Kenig, "The Relationship between Water Wetting and Ice Adhesion," Journal of Adhesion Science and Technology, Vol. 23, No. 15, 2009, pp. 1907-1915. doi:10.1163/016942409X12510925843078

[31] M. Zou, S. Beckford, R. Wei, C. Ellis, G. Hatton and M. A. Miller, "Effects of Surface Roughness and Energy on Ice Adhesion Strength," Applied Surface Science, Vol. 257, No. 8, 2011, pp. 3786-3792. doi:10.1016/j.apsusc.2010.11.149 